\title{
The City Centre Fifty Years On: Functional Regions in London Redefined from Taxi Flows
}

\author{
Jon Reades ${ }^{1,3}$, Urška Demšar², Ed Manley³ \& Michael Batty ${ }^{3}$ \\ ${ }^{1}$ Department of Geography, Kings College London (KCL), Strand, \\ London, WC2R 2LS, UK
}

${ }^{2}$ School of Geography \& Geosciences, University of St. Andrews, St. Andrews, KY16 9AJ, Scotland, UK

${ }^{3}$ Centre for Advanced Spatial Analysis (CASA),

University College London (UCL), 90 Tottenham Court Road,

London, W1T 4TJ, UK

Email addresses: jonathan.reades@kcl.ac.uk; urska.demsar@st-

andrews.ac.uk; ed.manley@ucl.ac.uk; m.batty@ucl.ac.uk.

\section{Key Words}

Principal Components Analysis (PCA),

Taxi Journeys,

Comparative Spatial Analysis,

Overlapping Zones,

Complex Spatial Structure,

London 


\section{Introduction}

\section{An historical comparison}

Fifty years ago, geographers first began to explore spatial patterns using rudimentary statistical methods that sought to extract the key determinants of spatial structure from a multitude of independent variables. Regression, correlation, principal components and factor analysis represented the cutting edge of quantitative geography. Since then there has been a veritable explosion in methods with many of these multivariate techniques being elaborated into schemes for searching for patterns in very large data sets through various strategies often referred to now as 'data mining'. Spatial data itself has also changed, becoming ever larger and, with the development of sensors that capture data in real time, complementing or even supplanting the manual surveys that were the dominant mode of collection in the past. Developments in computation have made all this possible and we now have at our disposal many 'big data' sets that require powerful methods developed over the last fifty years in their analysis. To an extent, we are in the midst of what Wyly (2014) has called a 'new quantitative revolution'.

It is always difficult, and often impossible, to compare what happened in the past with what is happening today, and there are very few comparative studies that attempt to chart changes in our understanding in this way. But in 1970, Transactions of the Institute of British Geographers published what we regard as a seminal article on detecting functional regions from a set of interactions (traffic volumes) between small partitions (zones) of a city centre using then state-of-the-art multivariate methods, specifically Principal 
Components Analysis (PCA) and Factor Analysis. Goddard (1970) used a 10\% sample of taxi journeys in Central London as recorded in the 1962 London Traffic Survey (LTS) to identify regions associated with major sources (origins) and sinks (destinations) of journeys. He suggested that Central London could be considered as an integrated set of subregions of different activity types that generate these trips. And while this kind of analysis has developed substantially over the last fifty years, the notion of identifying clusters of similar zones from common attributes associated with locations or patterns of interaction is still a major preoccupation of urban geography and spatial analysis (Roth et al. 2011; Reades and Smith 2014).

Our ability to compare what Goddard did with more contemporary approaches arises from a unique opportunity to compare his data and analysis with one built from mini-cab trips undertaken in 2011/12. Goddard's data came from logbooks completed by a sample of taxi drivers over one week in July 1962. Fifty years later, we have access to 1.3 million passenger journeys made over 12 weeks by the mini-cab firm Addison Lee (hereafter called AddLee). The data sets could not be more different: the LTS data is a simple sample for Central London, while the AddLee data is complete and covers all of Greater London. The 1962 data includes trips made by cruising for fares, as well as the usual fare-paying trips, while the AddLee data is for pre-booked trips only. The LTS data was coded to 70 traffic activity zones (TAZs) of which one was empty, while the AddLee data is accurate to a few metres, but has been aggregated to zones of a similar geography for comparative purposes. In a sense, everything about the approach is different, but the motivation for understanding spatial structure of a major city 
remains the same and, as we will see, the methods used in the 1960s remain surprisingly useful in a contemporary 'big data' context.

However, the inspiration for this work is not solely a comparison of old and new data, but also of the methods and outcomes of this analysis. Goddard's original work was clearly constrained by the computers of the day, and we thus ask the question 'does his approach remain relevant?' and 'what, if anything, about central London's functional spatial structure has changed in the intervening fifty years?' In fact, although we uncover some long-overlooked issues in the original work, we also find that his approach offers specific analytical advantages over more recent community detection methods borrowed from network science to associate flows to discrete regions (for a good overview of these see Farmer and Fotheringham 2011). Our results highlight the importance of this kind of spatial analysis to the geographical tradition; we find that PCA delivers superior results when the system of flows is highly polarized. In such cases, many contemporary 'big data' algorithms have difficulty effectively partitioning a set of nodes/zones into anything other than a simple core/periphery structure and thus fail to find meaningful structures in the data. Our approach, which is a contemporary realization of Goddard's (1970) attack on the problem, is in this case much more appropriate, implying that fifty years' worth of development in the field has not necessarily invalidated the approach set out in the 1970 paper.

\section{The wider context}

The use of transportation and communications flow data to derive regions rooted in functional interactions can be traced back to the rise of transportation 
planning in the 1950s (Voorhees 1955) and the increased accessibility of computers able to perform matrix analyses at speed (Berry 1964). While today's desktop and server systems may make some of the analytical challenges faced by these earlier researchers seem almost quaint, the basic question of how best to partition a set of zones into analytically useful regions remains germane. Indeed, our increased mobility and ability to interact at a distance makes the data-driven interrogation and derivation of regional structure more important than ever. While it is well-understood that different regionalisation methods can produce substantially different 'optimal' sets of smaller regions, what is less frequently noted is that many partitioning methods exclusively assign each input node or zone to a single region based on some concept of 'containment' or 'integration'. Typically, regions are defined such that intra-regional links are statistically more likely than inter-regional links. The two critical choices are: what metrics define the expectation of a link between two nodes, and what constraints govern permissible regions. For instance, the 'intramax' modelling of commuting flows not only seeks to ensure that the majority of trips are internal to a derived region, but also that the region is geographically coherent (Nielsen and Hovgesen 2008).

This exclusive association between zones (nodes) and regions (groups of nodes) can be a natural, and indeed powerful approach to understanding geography, and it is an idea that is well-suited to many analytical and administrative purposes. It has been used on a range of flow-based spatial analyses (Expert et al. 2011) but where there is strong evidence of polycentricity and where there are multiple specialised business centres generating and receiving large numbers of trips across a wide area (Taylor et al. 2006), it is 
worth questioning this type of exclusive partitioning. For example a recent example undertaken by Zhong et al. (2014) although appropriate for exclusive regional definitions is inappropriate for improving our ability to see overlapping flows where one zone contributes to multiple regions.

In the analysis that follows, we begin by outlining the methodology that Goddard (1970) used; we then introduce the idea that we can 'simplify' the raw flow data by identifying a new set of dimensions that are more parsimonious than the original ones. This kind of data reduction is a generic problem and we follow the method used by Goddard (1970) which consists of extracting the principal components from the data, thereby realigning the data set to produce a clearer picture of the importance of different origins and destinations to the shape of the 'data cloud'. This process then enables us to search for sets of origins and destinations of flows that form natural regions based on the similarity of flows between these sets of places. We speculate a little on the appropriateness of PCA, arguing that this technique has withstood the test of time, and then we consider the results, painting a picture of increasing complexity associated with overlapping functional regions in Central and Inner London.

\section{A methodology for exploring and extracting basic spatial dimensions}

\section{The old and the new data}

Goddard's LTS data was coded to 70 Traffic Analysis Zones (TAZs) created by the Office of Population, Census and Surveys from the 1961 Census. This data covers 
all types of trips, but most would have been generated spontaneously by 'hailing' a cab on the street. In contrast, the 2011 data set is based entirely on pre-booked trips from London's largest 'mini-cab' firm, Addison Lee (AddLee) which operates a fleet of more than 2,500 vehicles on a daily basis.

Figure 1: 1970 \& 2011 Traffic Analysis Zones comparison

a) 1970 zones from 1962 London Traffic Survey; b) 2011 Zones from London Traffic Model

a.

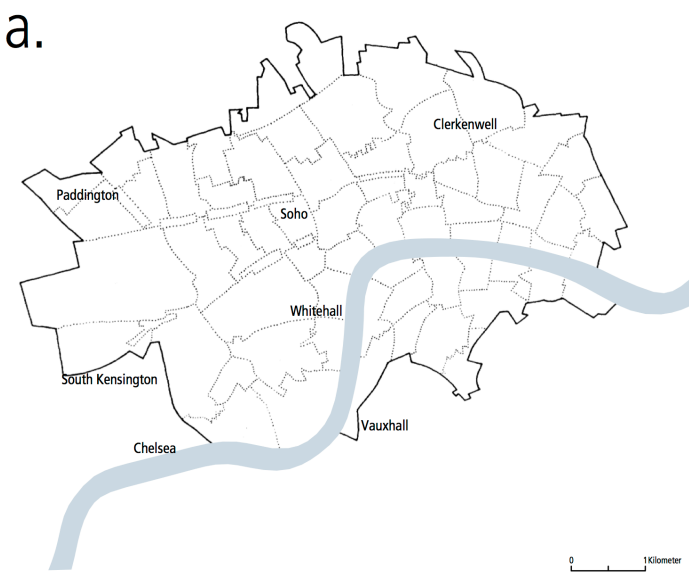

b.

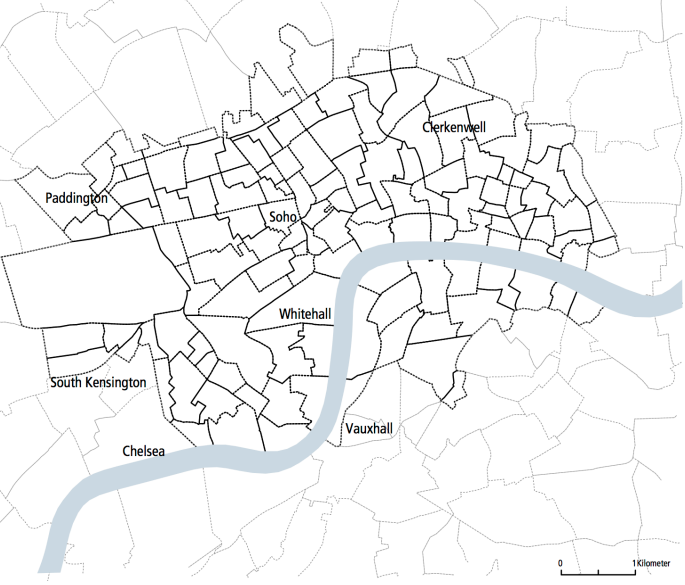

AddLee provided us with fleet movement data for all passenger travel occurring between $1^{\text {st }}$ December 2010 and 28 $8^{\text {th }}$ February 2011, enabling us to build a comprehensive $0 / \mathrm{D}$ (origin/destination) matrix for their operations in Greater London. Since the precision of GPS data theoretically enables us to identify address-level origins and destinations, the data were spatially aggregated so as to minimise the risk of re-identification (Zheleva and Getoor 2007). The aggregation was made to the current Traffic Analysis Zones used by Transport for London which we will define as $n$ origins $i=1,2, \ldots, n$ and $m$ destinations $j=1,2, \ldots, m$ and this generates a trip matrix $\left[T_{i j}\right]$ which constitutes the basic data for the comparison. Figure 1 shows the relevant historical and contemporary TAZ boundaries, highlighting the stability of these units within central London. We are therefore able to replicate Goddard's procedures using a 
broadly comparable set of spatial units, while also extending the analysis to the entire London metropolitan region, something that was not possible for Goddard.

The number of journeys recorded by Goddard (1970) is not known, but we estimate from his diagrams that the daily weekday average is of the order of 18,000 trips. From the AddLee data we get a similar weekday average of 19,000 journeys, although for the analysis we have used all weekday data over the 3 months, not an average. The volumes are roughly comparable, but in should be noted that the nature of the two services are potentially quite different because of the pre-booking aspect of AddLee's services. We constrained our analysis to weekdays on the basis - as Goddard also argued - that these were more likely to be work-related trips. Figure 2 provides an overview of the spatial and flow relationships: Figure 2(a) shows strong links emerging between the main business airports (Heathrow and London City) and employment zones in the Central Business District (CBD). And this highly polarised structure is reflected in the cumulative magnitude of the in- and out-flows which we compute as $D_{j}=\sum_{i} T_{i j}$ and $O_{i}=\sum_{j} T_{i j}$ shown in Figure 2(b).

Figure 2: Flows, origin and destination volumes

a) undirected volumes of AddLee trips between TAZs; $\underline{b}$ ) origin (Out-Flows) and destination (In-Flows) by TAZ illustrating the dominance of different destinations 
a.

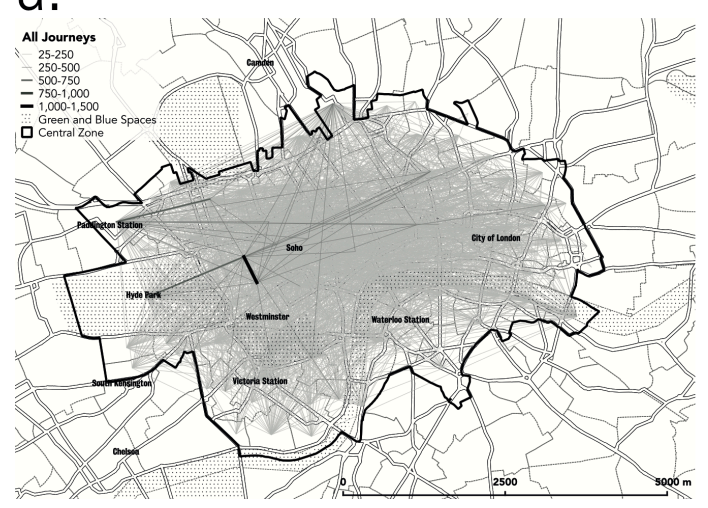

b.

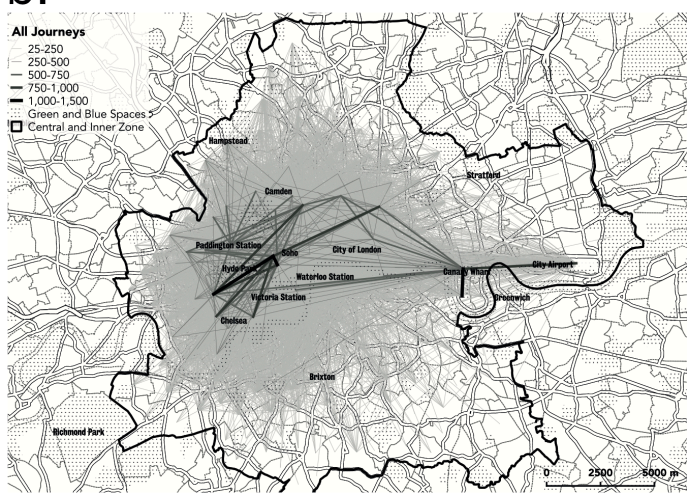

\section{Multivariate analysis using principal components}

Essentially, Goddard sought to identify the sources of variation in the flow data by extracting the key dimensions that accounted for most of the variation in the trips to different destinations. He used what was then a comparatively new method called Principal Components Analysis (PCA) which generates a new set of ordered dimensions oriented so as to better-align with the raw 'data cloud'. The importance of each of the new dimensions is connected to the amount of variation in the raw data that the dimension explains ${ }^{1}$. This 'importance measure' is referred to as a 'loading', and when the loadings are applied to an original data point we can compute a 'score' for each observation that reflects its importance to that dimension. The problem can thus be seen as one in which the original data set is transformed so that its structure becomes clearer, in this case with respect to the relative importance of the destinations which are defined by the loadings and the relative importance of the origins which are defined by scores. In fact, after transposition of the matrix PCA could also be used to derive

1 The technique goes back to work by Galton and Pearson (both at UCL) in the late 19th century. It was developed in a different context by Hotelling in the 1920s but it only became known once statistical packages were developed on main frame computers from the late 1950s. 
loadings for origins and scores for destinations, thus articulating the problem in a kind of dual way that we consider in more detail below.

Having extracted the scores and loadings from the data, Goddard went on to further adjust the axes by changing the variation explained with respect to the dimensions (varimax rotation); however, this made little difference to his results and the mathematical rationale for doing so is unclear ${ }^{2}$. Almost needless to say, after 45 years Goddard can no longer remember the precise details, and although PCA has subsequently become an important technique for dimensionality reduction and seeking patterns in multivariate data, it was in its infancy then and thus highly experimental at the time ${ }^{3}$.

${ }^{2}$ In fact, it would probably only make sense - and have any effect on the results - if what was actually performed was Factor Analysis, in which the number of dimensions is specified in advance, and not PCA. Varimax rotation (Kaiser 1958) is typically used to maximise the variance explained by each derived factor while also ensuring that, as far as possible, each one is correlated with only one of the original variables. Rotation of PCs is sometimes employed in PCA, particularly in atmospheric science (Joliffe 2002), but its validity is debatable since PCs derived via the correlation or covariance matrices already maximise variance. Hence, not only does rotation of the axes in the PC space risk changing the ordering associated with eigenvalues (Daultrey 1976), but the criterion of each rotated PC to be as closely bound as possible to a single initial variable (Harris 2001) is not very meaningful (Demšar et al. 2013). Having reviewed Goddard's (1970) results in detail, we suspect that varimax rotation was not actually performed in this way or was not performed in the way assumed by those operating the package. Consequently, we decided against rotation and performed our analysis on untransformed PCs.

${ }^{3}$ In 1969, John Goddard (personal communication) used a standard package for PCA which was stored on a set of punched cards to which the data were then attached on another set of cards. This package was available only on the IBM 7094 at Imperial College, so John had to take his data over to Imperial from the LSE on the Number 73 bus. Many times. The program was run in batch 
To briefly review PCA, let us define a data matrix $\left[X_{i j}\right]$ as having the same dimensions as the trip matrix $\left[T_{i j}\right]$. We distinguish the matrix $\mathbf{x}$ from $\mathbf{T}$ simply because this name change more closely relates our own exposition to the standard ones found in works on multivariate analysis. Anyway, $\mathbf{x}$ is formed from a set of $n$ observations of a set of $m$ variables. Thus each variable $j$ is a column of matrix $\left[X_{*_{j}}\right]$ and each row $i$ is a set of observations across each variable. The PCA problem can be stated very simply as one where we need to express $\mathbf{x}$ in terms of a new set of transformed axes in a $m \times m$ matrix $\mathbf{P}$ where each column of $\mathbf{P}$ is a principal component or dimension $\left[P_{j}\right]$. The linear transformation of $\mathbf{x}$ by $\mathbf{P}$ yields a new matrix $\mathbf{Y}$ and can be written in matrix form as $\mathbf{Y}=\mathbf{X} \mathbf{P}$; in full form this is

$$
Y_{i k}=\sum_{j} X_{i j} P_{j k}
$$

The new variables $Y_{i k}$ are the scores, while their contributions to the new axes $P_{j k}$ are loadings; consequently, $\mathbf{Y}$ is often called the score matrix and the $\mathbf{P}$ loading matrix.

The essence of PCA is to choose the new dimensions according to some reasonable criteria. The first criterion is that the new axes expressed by $\mathbf{P}$ are

mode with a turnaround of about 24 hours. John did not write the program, and was partly advised by a programming advisory service at the LSE, but he drew his inspiration from Rummel's (1967) classic monograph Understanding Factor Analysis. As users of such systems had little control over the program itself, it is not possible to reconstruct exactly what variant of PCA John used as all this information is now lost in the mists of time. 
orthogonal to one another, that is, they are independent of each other. The second is that these axes are chosen so that the covariance between the new variables $\mathbf{Y}$ given by the covariance matrix $\mathbf{C}_{Y}=[1 /(n-1)] \mathbf{Y} \mathbf{Y}^{T}$ is a diagonal matrix where all of the variation is between the new variables and there is zero covariance between each of the transformed variables. Using this covariance and the transformation in equation (1), and after a little algebra, the covariance matrix can be written $\mathbf{C}_{Y}=[1 /(n-1)] \mathbf{P} \mathbf{X} \mathbf{X}^{T} \mathbf{P}^{T}$, from which it is clear that the principal components of $\mathbf{x}$ are the eigenvectors, or columns of $\mathbf{P}$. As $\mathbf{C}_{Y}$ can be diagonalised, each diagonal value is equal to the eigenvalue or the variance of $\mathbf{x}$ along the $\mathbf{P}$ direction and the off-diagonal values - the covariances - are zero.

With PCA it is often possible to summarise the data using only a handful of new dimensions, far fewer than in the original data. The art of data reduction is thus to find decompositions of the variance-covariance matrix that provide a limited number of principal components and thereby summarise the data efficiently using only a few dimensions. We can order the eigenvalues by magnitude, and then normalise them by the sum - which is the trace of the covariance matrix $\mathbf{C}_{Y}$ - to give us the percentage of the variance 'explained' by each dimension. When the proportion of the variance given by each eigenvalue is computed, it is often possible to define a cut-off point where the cumulative increase in variation explained falls below, say, $1 \%$. At that point it is assumed that the additional components are largely noise.

\section{Applications to origin-destination flows}

PCA for spatial analysis was particularly popular in the 1960 s and 70 s as it was one of the first tools available to examine structure in large data sets and it was 
quickly picked up by quantitative geographers who recognised its power and ease of use. It was often employed in conjunction with clustering or crossclassification to group areas into regions on the basis of inter-zonal similarity (Berry 1964) and, although less common, a number of studies attempted to use it on flow data in transportation (Black, 1973), phone calls (Goddard 1973), and as in this application, taxi journeys (Goddard 1970). Goddard's work was purely for the origin-destination trips by black cabs in central London as shown in Figure 1 but the new AddLee vehicle flow data that we have enables us to look at a much wider spatial set of flows as implied in Figure 2. Our main comparison here is with the AddLee data grouped into a larger number of zones in the central area which we define as region $R_{C}$ consisting of the matrix $\left[T_{i j}^{C}\right]$ which is a $133 \times 133$ matrix but we have also developed the same kind of analysis for a wider region that we refer to as Central and Inner London $R_{I}$ where the matrix of flows $\left[T_{i j}^{I}\right]$ is $391 \times 391$ in size.

In keeping with the original work by Goddard, we take these matrices as inputs to PCA such that origins can be considered observations and destinations are treated as variables, and we also follow Goddard in choosing correlationbased PC. This can be thought of as a 'destination-based analysis'; however, from the matrix formulation above it is clear that $\mathbf{x}$ could be transposed to tackle an 'origin-based analysis' analysis. The 'dual' problem treats origins as observations and destinations as variables, providing a different picture of city's functional regions. There has been little explicit consideration of the different empirical implications of these primal and dual problems for trip analysis, though they are referred to as R-mode and Q-mode PCA in the wider field of multivariate 
analysis. This issue can also be linked to developments in spatial interaction and network theory where the primal and dual problems have been more directly considered (Batty 2013).

\section{Applications and comparisons: the black cab and Addison-Lee}

\section{data sets}

\section{Goddard's analysis}

From a contemporary standpoint, Goddard's (1970) choice of the number of principal components (PC) as $k=6$ seems fairly arbitrary, though it does reflect the computing constraints of the time; he says: "Examination of the eigenvalues showed that beyond the sixth, each additional component added little to the overall level of explanation" (Goddard 1970). We opted to take into account only those PCs that contributed more than $1 \%$ of the variance observed in the observation matrix $\mathbf{X}$. In the AddLee data, 17 PCs were retained for the Central Area and 9 PCs for the larger area (Central and Inner London).

The contribution of each PC in the AddLee data is illustrated in the screeplots in Figure 3: the rank of the retained principal component is shown on the $x$-axis and its contribution to total variance is given on the $y$-axis. What is most noticeable about these plots is that the contribution falls off quite rapidly and although this result is quite common in PCA, the magnitude of the first PC suggests that there is one dimension that dominates the data cloud.

Figure 3: Eigenvalues v rank for regions a) for the Central Region; b) for the Central and Inner Region, both using AddLee data 

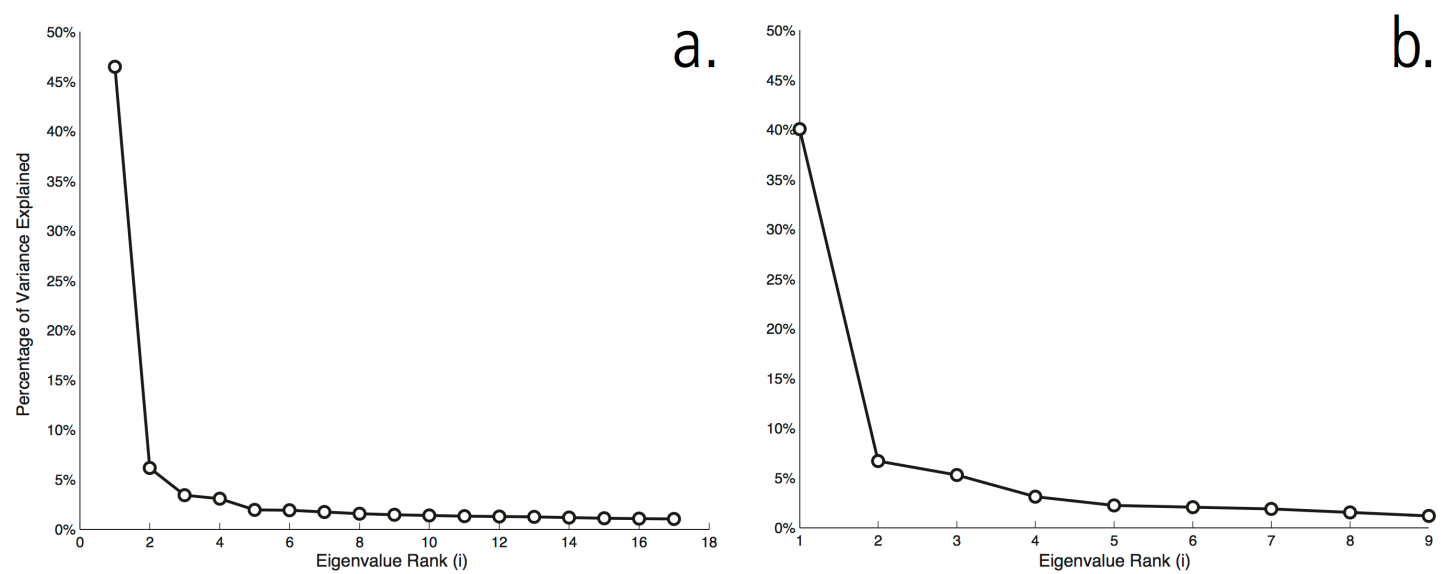

\section{Definition of functional regions from loadings and scores}

It is useful to turn again to the mathematics of PCA for a moment to help the reader to understand how we can use it to define regions. Recall that, in effect, we are rotating and rescaling the axes so that they align with the variance of the data cloud. To accomplish this transformation each variable in the new space becomes a linear combination of all of the attributes captured in each origin vector $X_{i k}, k=1,2, \ldots, m$ in the original space such that

$$
Y_{i k}=X_{i 1} P_{1 k}+X_{i 2} P_{2 k}+\ldots+X_{i j} P_{j k}+\ldots+X_{i m} P_{m k}
$$

Here $P_{j k}$ is the loading of attribute $X_{i j}$ on component score $Y_{i k}$. Because we have defined an O/D matrix with destinations as attributes, the largest absolute loadings correspond to the destinations that have the strongest effect on the variance associated with one dimension of the data cloud. The score is the data value in the new space of principal components, that is the transformed value of an observation in its $i^{\prime}$ th component - in this case an origin. The absolute score can be interpreted as the effect of a TAZ on one of the new dimensions, so a 
higher score indicates a greater contribution to the flows associated with that component.

In combination, the loadings and scores of the principal components that are retained are used to define regions as follows:

- important destinations which are those TAZs with the largest absolute loadings (i.e. those with highest $\left|P_{j k}\right|$ ). These are the destinations that have most influence (either positive or negative, depending on the sign) on a particular PC.

- important origins are those TAZs with largest absolute scores (i.e. those with highest $\left.\left|Y_{i k}\right|\right)$. These are the origins that are feeding the largest flows into a particular PC.

- functional regions are constituted from the union of important destinations with important origins.

Crucially, it should be noted it is entirely possible for TAZs to be important destinations and important origins simultaneously. In addition, the definition of 'important' will obviously have a significant impact on the partitioning into regions.

In Goddard's (1970) paper, importance was gauged using a manually calculated table of loadings and scores with cut-off values set to 1 for absolute loadings and to 0.5 for absolute scores. Again, these choices are based on the observed distribution of scores and loadings but they are, nonetheless, somewhat arbitrary (Goddard 1970). To put the method on a firmer ground, we have adopted principles from outlier analysis (Rogerson 2006): a TAZ was considered important if and only if the absolute value of its loading or score 
exceeded the value of the mean $\pm 1.5 \times$ the intra-quartile range of all absolute loadings or scores on a PC.

Figure 4: Distribution of origin loadings for Central Region $\left(R_{C}\right)$

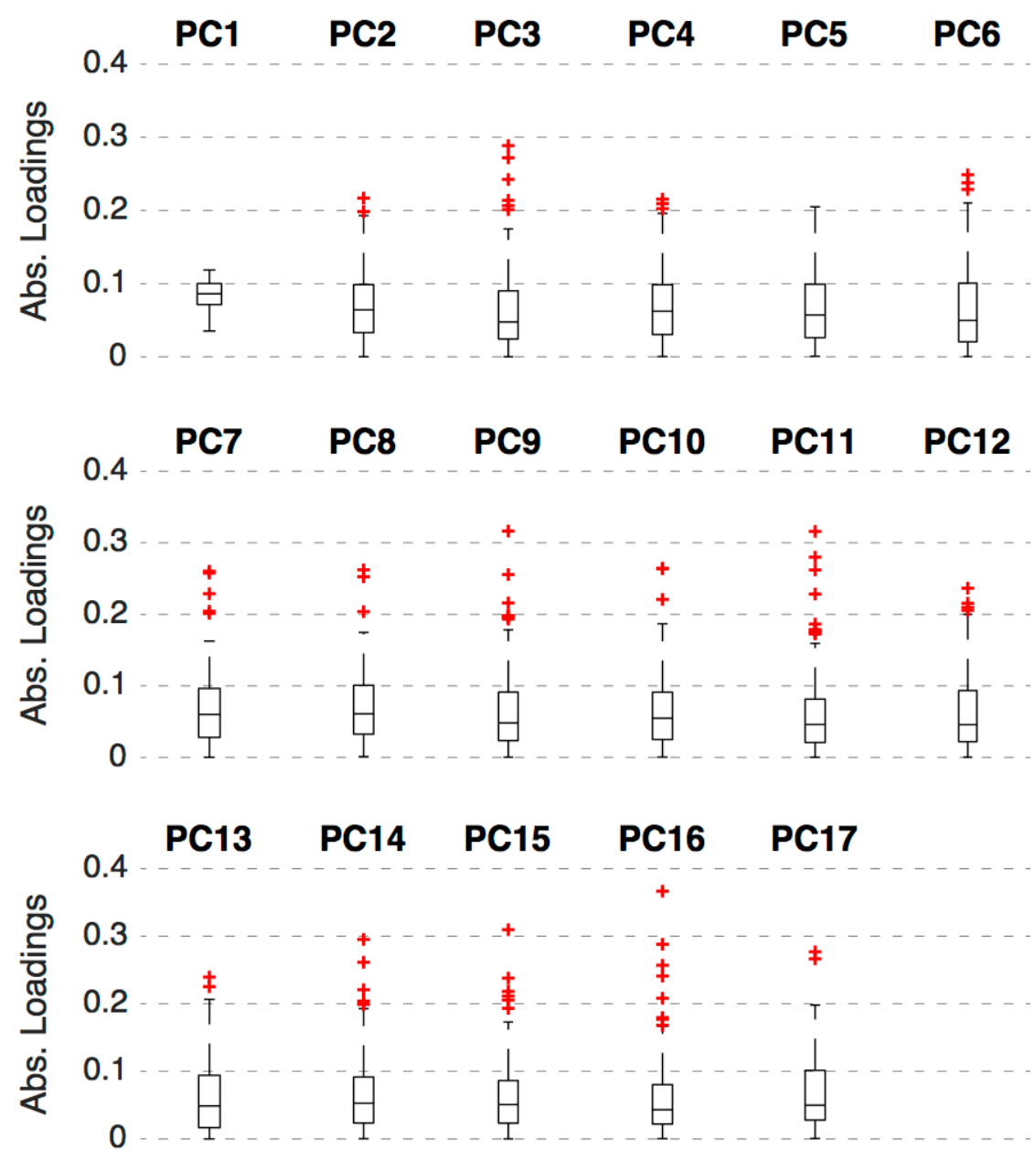

Figure 4 demonstrates how outlier-based selection of important values works for central London $R_{C}$ (the scores for $R_{C}$ and corresponding results for $R_{I}$ are available on the Supplementary Online Materials web site). The distribution of loadings for each PC is shown as a boxplot in Figure 4. All PCs, except the first, have a highly-skewed distribution of values, and this is indicated by the central box (which contains values between the upper and lower quartiles) being positioned towards the lower edge of the plot. The small crosses 
above the whisker are outliers, and in the case of the loadings they therefore represent the most important destinations. In order to define important destinations for PC1, which has no outliers, we investigated the results in more detail and found that selecting the top 5\% of loading values for PC1 yields a component of a similar size to the other PCs.

\section{Reworking and extending Goddard}

\section{Analysis and interpretation of activity hotspots in Central London}

We began this analysis by attempting to parallel Goddard's (1970) approach with a view to developing interpretations which were as close as possible to his original findings, and to this end, we did not first consider the full area covered by AddLee. However, a cursory glance at the raw data shown in Figure 2 and the results of the outlier analysis above already suggest a more much more highly

polarised system than was the case 50 years ago with many more long-distance flows. These findings make problematic an exclusive clustering approach based on the notion of non-overlapping regions, and thus suggest to us that PCA is useful to permit us to define an overlapping regionalisation of TAZs.

We now summarise our findings, highlighting the most important regions obtained from our analysis and relate these to Goddard's original study. Derived from PC1, Region 1 (shown in Figure 5a) has the densest interconnections between zones serving as important origins and destinations simultaneously. This result points strongly towards a 'core' for AddLee journeys within Central London, and Region 1 is the only grouping that has anything resembling contiguity. In terms of what the region actually represents, the origins are predominantly those of less-connected mainline rail stations to the East and 
North, and the destinations are predominantly in areas of luxury accommodation and consumption.

Figure 5: Central Region destinations: the two most important regional configurations

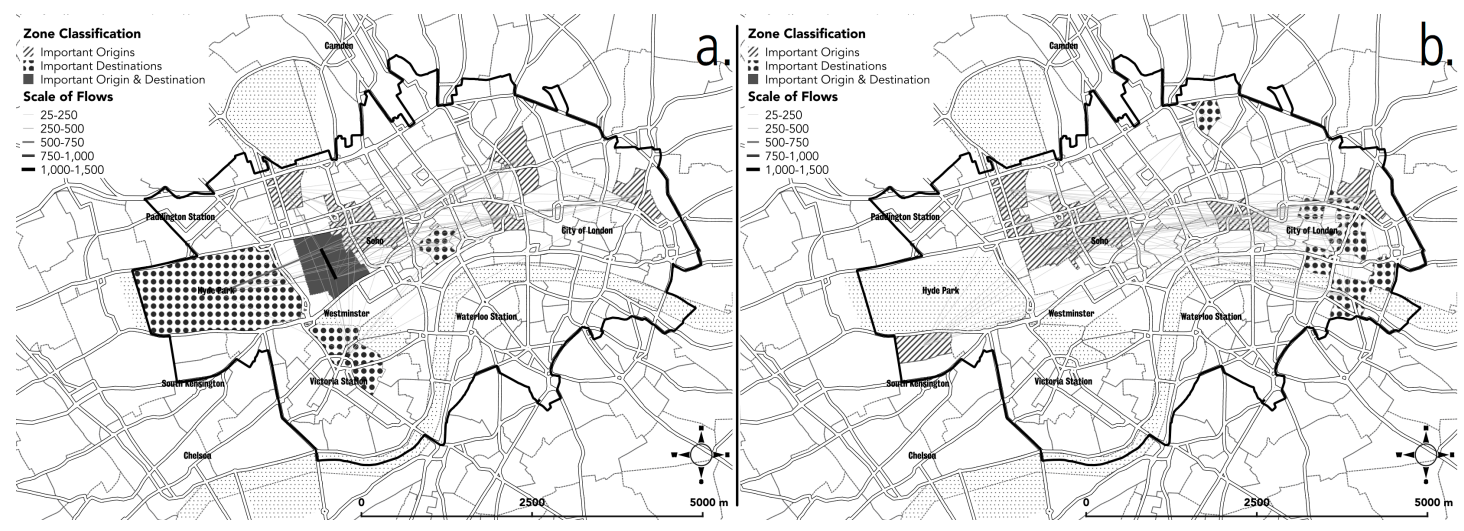

Figure $5 \mathrm{~b}$ demonstrates the value of this PCA -based approach: Region 2 contains several zones also selected for Region 1, but it is dominated by a strong east/west relationship based on predominantly residential origins to the west and destinations in the heart of the City or in the emerging agglomeration of consulting and related firms on the South Bank around London Bridge station. In fact, moving down through the remaining fifteen regions shows increased geographical polarisation across the board: together with increased dispersion, this points strongly towards a different spatial pattern from that of Goddard's black cabs.

Region 3 (Figure 6a) serves to highlight the usefulness of the ranking that falls naturally out of a PCA-based approach: we know that this region accounts for less variance than Regions $1 \& 2$ and can infer a 'narrower' type of trip pattern, one undertaken by fewer individuals. In principle, it is possible to disaggregate the journeys underpinning this cluster in order to betterunderstand them, but their spatial location points to non-work activity: a cluster 
of origins in Kensington \& Chelsea is loosely connected to King's Cross stationthis journey is not particularly attractive by Tube because of congestion-and to the South Bank. Region 4 captures flows from the northwest of Central London to Waterloo and London Bridge stations, another journey that is more pleasant if no faster or cheaper - by car.

Figure 6: more Central Region configurations

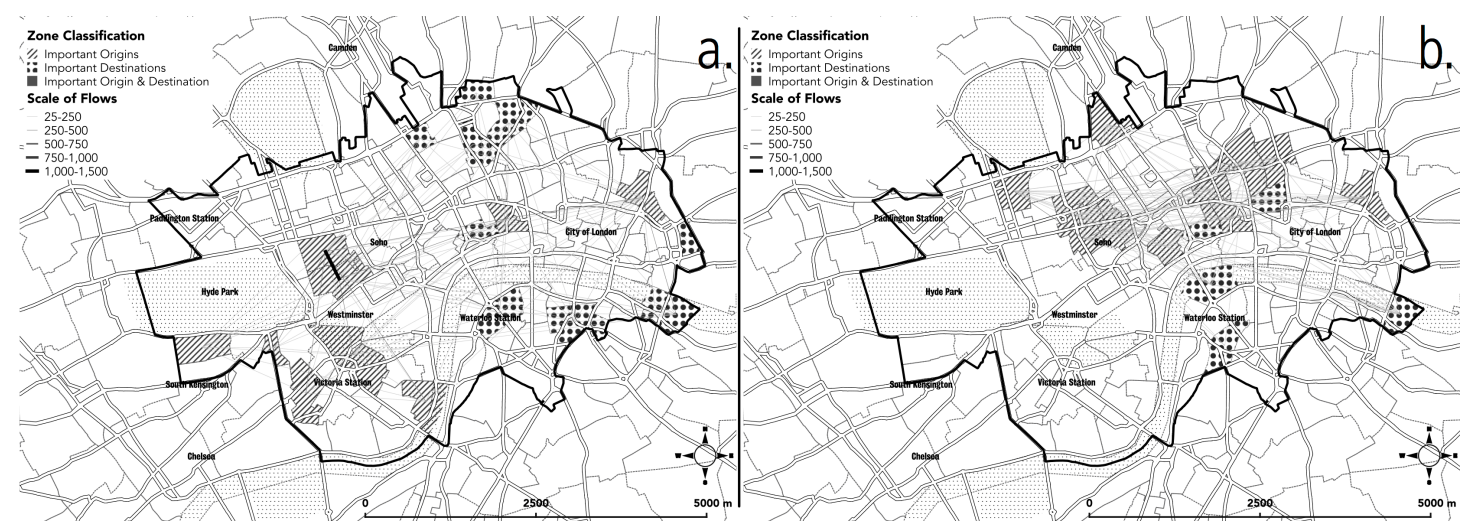

The remaining regions (available in the Supplementary Online Materials) each account for progressively less of AddLee's journeys-recall that the percent of variance explained can be found in Figure 3. The structure of these regions is, of course, influenced by factors that are not immediately evident from the map: Region 3 and 4, for instance, also join origins and destinations that are relatively weakly connected by London's Tube system; the routes may involve multiple transfers and congestion. Note, however, that the AddLee data reflects a particular set of behaviours, those of cash-rich, time-poor knowledge workers at the top end of London's income spectrum, and so these are not the same functional regions that would emerge from a study of, for instance, London's omnipresent bus system., It is essential that 'big data' be properly contextualised in order to understand what they reveal, and what they elide. 


\section{Extending the analysis to the wider city}

Turning now to the bigger picture, we will briefly interpret the larger functional geography of taxi flows as they pertain to both Central and Inner London. We cover this in more detail in our Supplementary Online Materials, but for completeness, and to extend Goddard's work, we review the top-level findings here. This scale of analysis was simply not possible in the 1960 s, so there is an interesting question of scale: are the patterns produced in the core reproduced at wider scales as well? Does the link between prestigious residential areas and major transit and employment sites hold, or is there a shift in usage such that functional regions are differently constituted?

In like-manner, for the Central \& Inner set of 391 zones we extracted the most important PCs, finding 9 that contributed more than $1 \%$ of variance. We show the relevant figures in the Supplementary Online Materials and will simply describe the spatial groupings here: as in our first analysis, the first region extracted constitutes an obvious functional cross-cutting 'core' for travel. However, it is notable that at this level there is significant 'in-filling': areas whose in-/out-flows were not significant when evaluating only Central London flows. The second region has an altogether different structure: while incorporating much of the first functional region as an origin, it has a strong east/west structure incorporating the City and London City Airport as well as, rather surprisingly, Croydon. Interestingly, for the remaining regions, many of their origins are zones that are currently poorly-served by high-speed links to Central London and to the airports; however, some of these 'routes' will be joined up from 2018 with the launch of the Crossrail 1 and, eventually, Crossrail 2 services. 
In a sense, we can see that London's structure is still very much monocentric: an extended CBD is the main draw for high-value journeys. The highest-value journeys and, by implication, the highest-value interactions are still dominated by 'Central London' even if the centre is rather larger than it was fifty years ago. Overspill of financial activity from the City of London does mean that hedge funds are now principally located in Mayfair and to the immediate southwest, while successful 'new economy' and business services firms can be found around the Old Street Roundabout (now rebranded as 'Tech City'), for instance.

However, a closer consideration of what is actually in 'the core' problematises this simplistic view: first, the size of the core is rather larger than we would have expected if it were a traditional style of CBD since it now incorporates a much wider range of functions-including entertainment, leisure, and residential activities-and second, the range of cross-cutting flows is more complex than a simple structure would imply. Within the CBD, the network of flows connects zones to mainline rail stations in much the same way that Goddard (1970) observed in the 1962 data, but it is now impossible to isolate each station as a 'source' or 'sink' for a given region. We also see the densely traversed core spreading westward along the Thames to include tech firms in previously overlooked areas such as Richmond and across the more northerly parts of South London. In short, the core's its persistence across these extracted regions is not purely a function of residential demand. 


\section{Reflection \& constraints}

As we have emphasised, in human geography it is rare to have the opportunity to revisit a research question and analysis from many years ago. The opportunity to do so highlights the fact that the analysis of functional regions not only remains relevant, but also that 'older' approaches can continue to shed light on current challenges. In this particular case, PCA offers benefits over more recent network-based approaches for dealing with highly polarised systems of flows, such as occurs in the AddLee data set. We can expect that in other big cities their centres will also be disproportionately large in comparison to their peripheries, and the degree of overall polarisation similarly extreme.

Of course, replication also poses some real challenges, and there are some important constraints that should also be noted here. Pre-booked mini-cabs obviously imply a degree of advanced planning for longer-distance journeys. AddLee's bookings have a strong business bias, enabling us to examine economic links directly. The relatively higher monetary and planning costs of mini-cab bookings mean that such journeys tend to be of higher value to the customer: in a city like London, mini-cabs are unlikely to be used to pop out to the shops, but they are common for trips to business meetings or to major events. In this sense, the extent to which AddLee data is 'socially constructed' (Johnston et al. 2014) works in favour here, highlighting the business usage that underpins the framing of our research as an analysis of the functional regions of the city.

However, the results presented above should not be presumed to provide substantive insight into more local interactions. Today, over short distances, people many walk, some cycle using a hired public bicycle and, for intermediatelength trips, some opt to take a black cab. In a contemporaneous related paper, 
Goddard (1973) himself found that one-third of business meetings in Central London were reached on foot. So although the addition of Canary Wharf has extended London's core in significant ways, we should not underestimate the continuing importance of physical proximity in a pedestrian context, particularly to 'high-touch' industries such as legal, finance, and culture.

The use of mini-cab data therefore implies a lack of direct comparability between our results and those of Goddard. However from a research standpoint, the importance of that difference may be more apparent than real: the prebooking requirement implies that we are capturing more formal interactions between large firms. We are therefore less likely to be sampling personal or tourist usage and so the results may be more formally relevant to a functional regional analysis. The AddLee data is therefore more appropriate for identifying the polycentric nature of London's centre than was Goddard's black cab data of 50 years ago. Planned journeys in the AddLee data can be linked to what Goddard (1975) called the 'programmed interactions' that occur at a relatively high level between strategic analysts from different organisations or different divisions within a large firm.

Analytically, we have shown that PCA offers several useful applications: when the concept of 'containment' is irrelevant, and when the structure of the network is highly polarised, PCA offers a way of generating overlapping communities when the number of communities is not known in advance. Of course, one challenge of this PCA process is that the 'behaviours' identified this way do not necessarily have direct, real-world correlates. However, this is not unique to PCA and other recent approaches to regionalisation (see Demšar et al. 2014) face the same challenge. In our case, each component accounts for a 
smaller and smaller part of the residual, which is to say the 'unexplained' portion of the model. There is a connection to 'revealed preference', but it is not direct: in a geographical context, this implies that PCA is most useful where the number of plausible regions is unknown but where the structure of the regions does not neatly tie back to any single set of 'causes'.

\section{Conclusions: an evaluation of comparative spatial analysis}

Embarking on this comparison, we quickly found dramatic differences between the kinds of data available now and what was available then. Clearly the nature of the journeys is different because taxi journeys in 1962 covered many more types of trips than those associated with a pre-booking operator. In one sense, companies like AddLee simply did not exist 50 years ago and so this type of journey could be considered a subset of those from 1962. In fact, we do not consider this to be the biggest source of difference between the two dates: changes in the behaviour of people using taxis is of greater import, but as is usual in comparing flow data of this kind, we have no data on the composition and purposes of journeys for either date. In general, all we can say is that it would appear that taxi journeys are considerably more common now with potential travellers considering many more opportunities for such journeys and many businesses being more geared up for such usage. To emphasise this point: UCL 
has an account with Addison Lee and some of the authors have used this service to travel both to airports and within the central area ${ }^{4}$.

It is also clear that activities driving travel within Central London have become considerably more complex during the last half century. This is reflected in the fact that there is a much greater diversity of hotspots - sub-centres which overlap in convoluted ways - than there were in 1962, and this is reflected too in everything from land values, house prices, volumes of traffic on various networks, capacity constraints on rail and subway systems, to pricing policies on parking to congestion. We have also shown that multivariate and network techniques that seek to partition complex flows into a hierarchy of sub-centres or clusters of nodes will have difficulty with a polarised network that yields from the exclusive assignments of nodes only a trivial core/periphery structure. PCA therefore appears to remain a robust technique for spatial partitioning and to be able to produce a much more convincing partitioning than can be generated using more recent and sophisticated techniques.

Interestingly, Goddard himself (1970, p.174) felt that an exclusive partitioning was desirable. There was a strong presumption at the time amongst quantitative geographers that their search was for contiguous and mutually exclusive partitions of space into a clear hierarchy of zones, notwithstanding the fact that Goddard did in fact consider that overlapping partitions were necessary for a full understanding of urban structure at this scale. The idea that unique

\footnotetext{
${ }^{4}$ If Goddard were doing this analysis now with 1969 technology but in a large contemporary UK university, he would be taking an Addison Lee cab down to Imperial College to run his computer programs, not the Number 73 bus!
} 
contiguous partitions are necessary for interpreting spatial structure has slowly weakened as geographers have accepted the notion that a hierarchy to spatial structure is no longer likely to be as clear cut, nor as desirable, as was assumed a generation or more ago. There is now a sense that cities benefit from structural complexity, and that societies have become more mobile and more able to interact in complex ways, such that the spatial structure of cities is increasingly fragmented into overlapping polycentric forms.

The process of polynucleation appears to have taken on special importance in London where districts are highly specialised and contribute to extensive cross-cutting transport and other flows (Hall and Pain 2006). Moreover results from smaller metropolitan areas, such as those in central Switzerland for example (Killer and Axhausen 2009), highlight the fact that overlapping commuting regions in which a single zone contributes to several regions at once may be integral to our understanding of the modern urban landscape. In short, our comparison of central London 50 years ago with today suggests that there should be a new focus on explaining how spatial structures are continuing to evolve in complex ways. In the quest to understand the future city, it appears that the tools introduced fifty years ago by researchers such as Goddard will continue to have an important role to play in our understanding of how networks of flows determine the structure of the contemporary city.

\section{Acknowledgements}

This work would not have been possible without the support of Addison Lee and Transport for London (TfL), and we would like to recognise their important contribution to this research. JR also wishes to acknowledge the 
support of the EPSRC (Grant \#EP/I018433/1) and of the European Commission (Complexity-NET/FP6 ERANET) during the early stages of this work; MB wishes to thank the European Research Council for support under 249393-ERC-2009AdG. 


\section{References}

Batty M 2013 The New Science of Cities The MIT Press, Cambridge MA

Berry B J L 1964 Approaches to regional analysis: a synthesis Annals of the Association of American Geographers 54 2-11

Black W R 1973 Toward a factorial ecology of flows Economic Geography 49 5967

Daultrey S 1976 Principal components analysis Concepts and Techniques in Modern Geography Geo Abstracts Ltd University of East Anglia, Norwich UK

Demšar $U$ and Harris $P$ and Brunsdon $C$ and Fotheringham $A$ and McLoone S 2013 Principal component analysis on spatial data: an overview Annals of the Association of American Geographers 103 106-128

Demšar U and Reades J and Manley E and Batty M 2014 Edge-based communities for identification of functional regions in a taxi flow network 8th International Conference on Geographic Information Science Vienna University of Technology, Austria

Expert P and Evans T S V D and Blondel V D and Lambiotte R 2011 Uncovering space-independent communities in spatial networks Proceedings of the National Academy of Sciences of the United States of America 108 7663-7668

Farmer C J Q and Fotheringham A S 2011 Network-based functional regions Environment and Planning A 43 2723-2741

Goddard J B 1970 Functional regions within the city centre: A study by factor analysis of taxi flows in Central London Transactions of the Institute of British Geographers 49 161-182 
Goddard J B 1973 Office linkages and location: a study of communications and spatial patterns in Central London Progress in Planning 1 109-232

Goddard J B 1975 Office Location in Urban and Regional Development Oxford University Press, Oxford UK

Hall P and Pain K eds 2006 The Polycentric Metropolis: Learning from Mega-City Regions in Europe Earthscan, London

Harris R J 2001 A Primer of Multivariate Statistics Lawrence Erlbaum Associates Publishers Mahwah, NJ

Johnston R and Harris R and Jones $\mathrm{K}$ and Manley D and Sabel C E and Wang W W 2014 One step forward but two steps back to the proper appreciation of spatial science Dialogues in Human Geography 4 59-69

Joliffe I T 2002 Principal Component Analysis Springer, New York

Kaiser F H 1958 The varimax criterion for analytic rotation in factor analysis Psychometrika 23 187-200

Killer V and Axhausen K W 2009 Mapping overlapping commuting areas Arbeitsberichte Verkehrs-und Raumplanung 555 Institute for Transport Planning and Systems (IVT) ETH Zurich, CH

Nielsen T A S and Hovgesen H H 2008 Exploratory mapping of commuter flows in England and Wales Journal of Transport Geography 16 90-99

Reades J and Smith D A 2014 Mapping the 'space of flows': The geography of global business telecommunications and employment specialization in the London mega-city-region Regional Studies 48 105-126

Rogerson P A 2006 Statistical Methods for Geography: A Student's Guide Sage London 
Roth C and Kang S M and Batty M and Barthelemy M 2011 Structure of urban movements: Polycentric activity and entangled hierarchical flows PLoS ONE 6 e15923. doi: 10.1371/journal.pone.0015923

Rummel R J 1967 Understanding Factor Analysis Social Science Research Institute Department of Political Science University of Hawaii Honolulu HI

Taylor P J and Evans D and Pain K 2006 Organisation of the polycentric metropolis: corporate structures and networks in Hall $\mathbf{P}$ and Pain $\mathbf{K}$ eds The Polycentric Metropolis: Learning from Mega-City Regions in Europe Earthscan, London

Voorhees A M 19552013 A general theory of traffic movement (The 1955 ITE Past Presidents Award paper) Transportation 40 1105-1116.

Wyly E 2014 The new quantitative revolution Dialogues in Human Geography 4 $26-38$

Zheleva E and Getoor L 2007 Preserving the privacy of sensitive relationships in graph data Proceedings of the 1st ACM SIGKDD International Conference on Privacy, Security, and Trust in KDD 153-171

Zhong C and Arisona S M and Huang X and Batty M and Schmitt G 2014 Detecting the dynamics of urban structure through spatial network analysis International Journal of Geographical Information Science 28 21782199 